\title{
More exact solutions to Waring's problem for finite fields
}

\author{
by \\ KeiJo Kononen (Oulu)
}

1. Introduction. Let $\mathbb{F}_{q}$ be a finite field of $q$ elements. For a positive exponent $k$, Waring's problem for $\mathbb{F}_{q}$ is the question of how many summands $n$ are minimally needed to express every element of $\mathbb{F}_{q}$ in the form

$$
\sum_{i=1}^{n} x_{i}^{k}
$$

where $x_{i} \in \mathbb{F}_{q}$ for all $i$. We define the Waring function $g(k, q)$ to be that minimal number of summands.

We shall prove the following results.

THEOREM 1.1. Let $m$ be a positive integer and $p, r$ primes such that $p$ is a primitive root modulo $r^{m}$. Then

$$
g\left(\frac{p^{\varphi\left(r^{m}\right)}-1}{r^{m}}, p^{\varphi\left(r^{m}\right)}\right)=\frac{(p-1) \varphi\left(r^{m}\right)}{2},
$$

where $\varphi$ is Euler's phi-function.

THEOREM 1.2. Let $m$ be a positive integer and $p, r$ odd primes such that $p$ is a primitive root modulo $r^{m}$. Then

$$
g\left(\frac{p^{\varphi\left(r^{m}\right)}-1}{2 r^{m}}, p^{\varphi\left(r^{m}\right)}\right)= \begin{cases}r^{m-1}\left\lfloor\frac{p r}{4}-\frac{p}{4 r}\right\rfloor & \text { if } r<p, \\ r^{m-1}\left\lfloor\frac{p r}{4}-\frac{r}{4 p}\right\rfloor & \text { if } r \geq p .\end{cases}
$$

We note that Theorems 1.1 and 1.2 generalize Theorems 1.2 and 1.3 respectively of [3], which cover the case $m=1$. A prime $p$ is a primitive root modulo $r^{m}$ for every $m \in \mathbb{Z}_{+}$if $p$ is a primitive root modulo $r^{2}$ and $r$ is an odd prime (see [2, Theorem 9.10]). This makes it rather easy to find primes

2010 Mathematics Subject Classification: Primary 11P05.

Key words and phrases: cyclotomic polynomial, equations over finite fields, exact values, Waring's problem. 
$p, r$ satisfying our assumptions for all values $m \in \mathbb{Z}_{+}$. For example a prime $p$ is a primitive root modulo $3^{m}$ for all $m \in \mathbb{Z}_{+}$if and only if $p \equiv 2(\bmod 9)$ or $p \equiv 5(\bmod 9)$.

2. Proof of Theorem 1.1. Put $t=r^{m-1}, q=p^{\varphi\left(r^{m}\right)}$ and $k=$ $(q-1) / r^{m}$. Let $\gamma$ be a primitive element of the finite field $\mathbb{F}_{q}$ and denote $\zeta=\gamma^{k}$. Then $\zeta$ is a primitive $r^{m}$ th root of unity and all the nonzero $k$ th powers in the field $\mathbb{F}_{q}$ are $1, \zeta, \ldots, \zeta^{m}-1$. Since $p$ is a primitive root modulo $r^{m}, \mathbb{F}_{q}$ is in fact the smallest extension field of $\mathbb{F}_{p}$ containing $\zeta$. Thus $\mathbb{F}_{q}=$ $\mathbb{F}_{p}(\zeta)$ and the minimal polynomial of $\zeta$ is the $r^{m}$ th cyclotomic polynomial $\Phi_{r^{m}}(x)=\Phi_{r}\left(x^{t}\right)=x^{(r-1) t}+\cdots+x^{t}+1$ (see for example [1, p. 65]).

We shall use the brief notation $\mathbb{Z}_{n}:=\mathbb{Z} / n \mathbb{Z}$ for the integers modulo $n$. A vector $\boldsymbol{a}=\left(a_{0}, \ldots, a_{r^{m}-1}\right) \in \mathbb{Z}_{p}^{r^{m}}$ will be called a representation for an element $a \in \mathbb{F}_{q}$ if

$$
a=\sum_{i=0}^{r^{m}-1} a_{i} \zeta^{i}
$$

Since $\mathbb{F}_{q}=\mathbb{F}_{p}(\zeta)$, every element $a \in \mathbb{F}_{q}$ has such a representation. Let $\bar{x}$ denote the smallest nonnegative integer in the equivalence class $x \in \mathbb{Z}_{p}$ for any given class $x$. Obviously, we may then represent $a$ as a sum of $k$ th powers in such a way that there are $\bar{a}_{i}$ summands $\zeta^{i}$ for every $i$ and the total number of summands is

$$
\|\boldsymbol{a}\|_{1}=\sum_{i=0}^{r^{m}-1} \bar{a}_{i} .
$$

Using the terminology introduced in [3] we call a vector $\boldsymbol{a} \in \mathbb{Z}_{p}^{r^{m}}$ admissible (with respect to \|\|$_{1}$ ) if $\|\boldsymbol{a}\|_{1} \leq\|\boldsymbol{b}\|_{1}$ whenever $\boldsymbol{b}$ is a representation for the same element $a \in \mathbb{F}_{q}$ as $\boldsymbol{a}$. The solution $g(k, q)$ for Waring's problem will now be the maximal value $\|\boldsymbol{x}\|_{1}$ for an admissible vector $\boldsymbol{x} \in \mathbb{Z}_{p}^{r^{m}}$.

A vector $\boldsymbol{b}=\left(b_{0}, \ldots, b_{r^{m}-1}\right)$ is a representation for the same element $a$ as a vector $\boldsymbol{a}$ if and only if there exist $c_{0}, \ldots, c_{t-1} \in \mathbb{Z}_{p}$ satisfying the equation

$$
\sum_{i=0}^{r^{m}-1} a_{i} x^{i}=\sum_{i=0}^{r^{m}-1} b_{i} x^{i}+\Phi_{r^{m}}(x) \sum_{j=0}^{t-1} c_{j} x^{j}=\sum_{i=0}^{r^{m}-1} b_{i} x^{i}+\sum_{j=0}^{t-1} c_{j} x^{j} \Phi_{r^{m}}(x)
$$

in the polynomial ring $\mathbb{Z}_{p}[x]$. Here

$$
x^{j} \Phi_{r^{m}}(x)=x^{j}+x^{t+j}+\cdots+x^{(r-1) t+j}
$$

for every $j=0, \ldots, t-1$. We define subvectors $\boldsymbol{a}^{(0)}, \ldots, \boldsymbol{a}^{(t-1)} \in \mathbb{Z}_{p}^{r}$ by the equations $\boldsymbol{a}^{(j)}=\left(a_{j}, a_{t+j}, \ldots, a_{(r-1) t+j}\right)$ and similarly for $\boldsymbol{b}$. Also, let $\boldsymbol{e}=(1, \ldots, 1) \in \mathbb{Z}_{p}^{r}$. Notice that a vector $\boldsymbol{y} \in \mathbb{Z}_{p}^{r}$ (i.e. in the case $m=1$ ) 
is admissible if and only if $\|\boldsymbol{y}\|_{1} \leq\|\boldsymbol{y}+z \boldsymbol{e}\|_{1}$ for every $z \in \mathbb{Z}_{p}$. The vectors $\boldsymbol{a}, \boldsymbol{b} \in \mathbb{Z}_{p}^{r^{m}}$ represent the same element if and only if all subvectors satisfy $\boldsymbol{a}^{(i)} \equiv \boldsymbol{b}^{(i)}(\bmod (\boldsymbol{e}))$, where $(\boldsymbol{e})$ denotes the submodule generated by $\boldsymbol{e}$ in the free module $\mathbb{Z}_{p}^{r}$.

It follows that $\boldsymbol{a} \in \mathbb{Z}_{p}^{r^{m}}$ is admissible if and only if each subvector $\boldsymbol{a}^{(i)} \in \mathbb{Z}_{p}^{r}$ is admissible. Moreover, the maximal norm for an admissible vector in $\mathbb{Z}_{p}^{r}$ is $(p-1)(r-1) / 2$ by [3, Theorem 2.5]. Thus the maximal norm for an admissible vector $\boldsymbol{a} \in \mathbb{Z}_{p}^{r^{m}}$ is achieved precisely when all the subvectors are admissible in $\mathbb{Z}_{p}^{r}$ of maximal norm, and it equals

$$
\|\boldsymbol{a}\|_{1}=\sum_{i=0}^{t-1}\left\|\boldsymbol{a}^{(i)}\right\|_{1}=t \cdot \frac{(p-1)(r-1)}{2} .
$$

3. Proof of Theorem 1.2. We shall use the notations from the previous section. Now all the $(k / 2)$ th powers in the field $\mathbb{F}_{q}$ are $0, \pm 1, \pm \zeta, \ldots, \pm \zeta^{r^{m}-1}$. Suppose $a \in \mathbb{F}_{q}^{*}$ is written as a sum of $(k / 2)$ th powers in the form

$$
a=\sum_{i=0}^{r^{m}-1} a_{i}^{(+)} \zeta^{i}+\sum_{i=0}^{r^{m}-1} a_{i}^{(-)}\left(-\zeta^{i}\right),
$$

where $a_{i}^{(+)}, a_{i}^{(-)} \in \mathbb{Z}_{p}$ for every $i$. Putting $a_{i}=a_{i}^{(+)}-a_{i}^{(-)} \in \mathbb{Z}_{p}$ we again get a representation of the form 2.1. The corresponding vector $\boldsymbol{a}=\left(a_{0}, \ldots, a_{r^{m}-1}\right) \in \mathbb{Z}_{p}^{r^{m}}$ will again also be called a representation for $a$. For every $x \in \mathbb{Z}_{p}$ put

$$
|x|=\min \{\bar{x}, p-\bar{x}\} .
$$

There exist $p$ different choices of $a_{i}^{(+)}$and $a_{i}^{(-)}$that lead to the same value $a_{i}$. Among these choices the smallest possible total number of summands $\pm \zeta^{i}$ is $\left|a_{i}\right|$. To see this, note that if we choose $a_{i}^{(-)}$arbitrarily then $a_{i}^{(+)}=a_{i}+a_{i}^{(-)}$ and

$$
\bar{a}_{i}^{(+)}+\bar{a}_{i}^{(-)}= \begin{cases}\bar{a}_{i}+\bar{a}_{i}^{(-)}+\bar{a}_{i}^{(-)} \geq \bar{a}_{i} & \text { if } 0 \leq \bar{a}_{i}^{(-)}<p-\bar{a}_{i}, \\ \bar{a}_{i}+\bar{a}_{i}^{(-)}-p+\bar{a}_{i}^{(-)} \geq p-\bar{a}_{i} & \text { if } p-\bar{a}_{i} \leq \bar{a}_{i}^{(-)}<p .\end{cases}
$$

So instead of the norm $\|\boldsymbol{a}\|_{1}$ we are interested in the so-called Lee norm

$$
\|\boldsymbol{a}\|_{2}=\sum_{i=0}^{r^{m}-1}\left|a_{i}\right|
$$

Again the solution of Waring's problem $g(k / 2, q)$ will be the maximal norm of an admissible element; the only difference is that "admissible" is now with respect to the Lee norm \|\|$_{2}$. The rest of the proof goes as before: again $\boldsymbol{a}$ is admissible if and only if each subvector $\boldsymbol{a}^{(i)}$ is admissible and according 
to [3, Theorem 2.6] the maximal Lee norm of an admissible vector of $\mathbb{Z}_{p}^{r}$ is

$$
g\left(\frac{p^{r-1}-1}{2 r}, p^{r-1}\right)= \begin{cases}\left\lfloor\frac{p r}{4}-\frac{p}{4 r}\right\rfloor & \text { if } r<p, \\ \left\lfloor\frac{p r}{4}-\frac{r}{4 p}\right\rfloor & \text { if } r \geq p .\end{cases}
$$

\section{References}

[1] R. Lidl and H. Niederreiter, Finite Fields, 2nd ed., Encyclopedia Math. Appl. 20, Cambridge Univ. Press, Cambridge, 1997.

[2] K. H. Rosen, Elementary Number Theory and Its Applications, 4th ed., AddisonWesley, Reading, MA, 2000.

[3] A. Winterhof and C. van de Woestijne, Exact solutions to Waring's problem for finite fields, Acta Arith. 141 (2010), 171-190.

Keijo Kononen

Department of Mathematical Sciences

University of Oulu

P.O. Box 3000

FIN-90014 Oulun yliopisto, Finland

E-mail: keijo.kononen@oulu.fi 